Corsino Rey

James D. Fortenberry

\title{
Prognostic markers for pediatric septic shock: which ones, when, and how?
}

Received: 1 June 2013

Accepted: 11 July 2013

Published online: 31 July 2013

(C) Springer-Verlag Berlin Heidelberg and ESICM 2013

C. Rey ( $)$

Pediatric Intensive Care Unit, Hospital Universitario Central Asturias, Oviedo, Spain

e-mail: corsino.rey@sespa.princast.es

C. Rey

Department of Medicine, University of Oviedo, Oviedo, Spain

J. D. Fortenberry

Pediatric Intensive Care Unit, Children's Healthcare of Atlanta, Atlanta, GA, USA

J. D. Fortenberry

Emory University School of Medicine, Atlanta, GA, USA

Septic shock remains an important cause of pediatric mortality. A reliable marker of eventual outcome of highrisk children in a pediatric intensive care unit within the first $24 \mathrm{~h}$ from admission would serve as a valuable clinical tool. Reliable prognostic markers for pediatric septic shock could improve clinical management based on therapeutic endpoints and stratify patients for more effective clinical research purposes. However, to date, in the search for these markers, many questions remain to be answered: Which available biomarkers provide practical, sensitive, and specific prediction? When is the optimal time to measure them? How can physicians best analyze marker results?

Regarding the first question, two categories of biomarkers exist (Table 1): those currently employed in research settings, and current clinically available markers. Relevant to the first group is the recent report from Kaplan and Wong [1] describing the development of the
PediatRic Sepsis biomarkEr Risk modEL (PERSEVERE) using 15 candidate stratification biomarkers. PERSEVERE is intended to predict outcome and illness severity for individual children with septic shock. Flow-cytometry-based inflammatory cytokine measurement [2] and other multimarker-based stratification strategies are also active areas of investigation.

As we await definitive applications of these promising innovative investigations, optimization in the management of clinically available markers also deserves clinical research. We understand that current markers for septic shock are limited in their utility, but the possibility exists to optimize their use by better answering the questions of when to measure and how to analyze. The great majority of research evaluating serum lactate as an indicator of tissue hypoxia has been conducted in adults. Recommendations regarding measurement of initial and subsequent lactate levels are included in the adult-directed Surviving Sepsis Campaign bundles [3]. Barker et al. [4] found that serial determinations of blood lactate levels were better predictors of death, because the duration of lactic acidosis was more important than the initial lactate value. Few studies have been performed in children, and results are controversial. Serum lactate levels were first noted to be higher in children with septic shock who had higher mortality $[5,6]$. However, other studies found that lactate could be increased by other stimuli independent of cellular hypoxia. For that reason, pediatric management in the 2012 Surviving Sepsis Campaign bundles still does not strongly recommend titrating therapy to lactate levels.

Given the current lack of available effective new markers for septic shock though, it is reasonable to rethink the use of easily obtained markers such as lactate in novel approaches for children. To that end, in the current issue of Intensive Care Medicine, Kim et al. [7] describe an innovative approach: the concept of lactate area as a potentially feasible and clinically useful predictor of mortality in pediatric septic shock patients. 
Table 1 Biomarkers utilized in clinical and research settings in pediatric septic shock, and information provided

\begin{tabular}{|c|c|}
\hline Biomarkers currently used in clinical setting & Information \\
\hline Lactate & Prognosis, possible therapeutic decisions? \\
\hline C-reactive protein & Diagnosis, antibiotic guide in neonates \\
\hline Procalcitonin & Diagnosis, prognosis, antibiotic guide \\
\hline Cytokines (interleukin-6, interleukin-8) & Diagnosis, prognosis \\
\hline \multicolumn{2}{|l|}{ Biomarkers currently used in research setting (examples) for potential clinical use } \\
\hline Neutrophil and monocyte markers (CD 64, CD 163, CD 11b, CD 15s) & Diagnosis \\
\hline Triggering receptor expressed on myeloid cells- 1 & Diagnosis \\
\hline Cytokines (tumor necrosis factor-alpha, interleukin-18) & Diagnosis, prognosis \\
\hline Lipopolysaccharide-binding protein & Diagnosis \\
\hline $\begin{array}{l}\text { Vasoactive peptides (pro-adrenomedullin, pro-endothelin, pro-atrial } \\
\text { natriuretic peptide, copeptin, etc.) }\end{array}$ & Prognosis \\
\hline $\begin{array}{l}\text { Endothelial/leukocyte adhesion molecules [soluble intercellular adhesion } \\
\text { molecule (ICAM)-1, vascular cell adhesion molecule (VCAM)-1, E-selectin] }\end{array}$ & Prognosis \\
\hline PERSEVERE model [1] candidate biomarkers: & Diagnosis, prognosis \\
\hline \multicolumn{2}{|l|}{$\mathrm{C}-\mathrm{C}$ chemokine ligand 3 and 4 (MIP-1alpha) } \\
\hline \multicolumn{2}{|l|}{ Neutrophil elastase 1} \\
\hline \multicolumn{2}{|l|}{ Fibrinogen-like 2} \\
\hline \multicolumn{2}{|l|}{ Granzyme B } \\
\hline \multicolumn{2}{|l|}{ Heat-shock protein $70 \mathrm{kDa} 1 \mathrm{~B}$} \\
\hline \multicolumn{2}{|l|}{$\begin{array}{l}\text { IL-1 } \alpha \\
\text { IL- } 8\end{array}$} \\
\hline IL-8 & \\
\hline \multicolumn{2}{|l|}{ Lipocalin 2 (NGAL) } \\
\hline \multicolumn{2}{|l|}{ Lactotransferrin } \\
\hline \multicolumn{2}{|l|}{ Matrix metallopeptidase 8} \\
\hline \multicolumn{2}{|l|}{ Orosomucoid 1} \\
\hline \multicolumn{2}{|l|}{ Resistin } \\
\hline \multicolumn{2}{|l|}{ Sulfatase 2} \\
\hline Thrombospondin 1 & \\
\hline
\end{tabular}

Kim's model describes "lactate area" to more broadly encompass lactate levels, clearance, and elevation in a summative fashion. They demonstrate that lactate area has stronger predictive value for mortality in pediatric septic shock patients than the single initial lactate level. Isolated lactate levels represent only current patient status. Lactate clearance adds some additional information, but describes only the change of marker levels as a percentage. The mathematical trapezoid method they employ to calculate lactate area provides a broader mean measurement over time that includes both the severity and duration of hyperlactatemia. Each trapezoid is obtained by calculating the area as the mean of two consecutive lactate values multiplied by the number of hours between them, providing a measure that includes the sum of trapezoids during the $24 \mathrm{~h}$ following admission. The trapezoid approach potentially provides more information than a single static lactate value, and is more indicative of dynamic lactate changes over the critical first $24 \mathrm{~h}$ of assessment and therapy, rather than an arbitrarily chosen time of first measure.

Kim's analysis has several limitations, including the retrospective approach, the relatively high mortality in the patient series, and the high numbers of chronic and hematologic/oncologic patients in the series. It most notably is limited by its small numbers. While obtaining larger numbers of patients in pediatric shock studies is difficult, further work is necessary to better characterize the lactate area in broader pediatric populations. Additionally, the lactate area method cannot distinguish patients with decreasing lactate concentration from those with increasing lactate concentration over a 24 -h period. Prognosis would be expected to be better in those with decreasing concentrations over time; this directional change should also be taken into account when interpreting serial lactate measurements.

Using early and repeated measurements to obtain an area calculated by the trapezoidal rule could improve prognostic value not only for lactate but also for other currently available markers. C-reactive protein has been historically used as a helpful marker of infection. Procalcitonin (PCT) has been recognized to have utility over recent years, to determine sepsis diagnosis [8], for severity classification of patients [9], and to guide antibiotic treatment duration [10]. Maintained elevated PCT values over a period of time identify a worsening of the inflammatory process due to the lack of septic shock control. Interleukin-8 can also predict outcome in pediatric septic shock with high negative but unacceptably low positive predictive values for mortality [11]. Other currently available clinical markers such as vasopressin and copeptin were not found to have utility for severity and outcome prediction in pediatric septic shock [12]. Combinations of these current clinically available markers could potentially help in prognostic stratification of pediatric septic shock estimating the risk of death. 
Individually, lactic acid as a marker of cellular hypoxia, PCT as a marker of the inflammatory process from infection and/or shock, and coagulation times [13] as markers of hypocoagulability can provide useful information during the early phase of septic shock. Employed in combination, and with novel trapezoidal measurements, these markers could be used to help in the assessment, prognostic, and decision-making processes.

Members of the International Sepsis Forum [14] have recommended greater standardization of biomarker methodologies, greater methodological rigor in biomarker studies, and wider integration of biomarkers into clinical studies, as well as increased collaboration amongst investigators, pharmaceutical and biomarker industry partners, and regulatory agencies. This recommendation comes with the realization that such efforts will require an unprecedented degree of systematic investigation and collaboration.

In summary, multimarker-based risk models for pediatric septic shock under investigation, including modalities of investigation in genomics, proteomics, lipidomics, and metabolomics [15], could represent the future in providing better clinical sepsis biomarkers. In the interim, we need to improve the use of currently available bedside clinical markers to enhance prognostic assessment of children with septic shock. A number of current clinical and research biomarkers have been evaluated specifically in children for pediatric septic shock (Table 1). Kim et al. have provided promising research, introducing a novel approach for use of early serial lactate measurements to stratify prognosis and subsequent monitoring of therapeutic effectiveness in pediatric patients with septic shock. Other promising available clinical markers of inflammation, infection, shock, and coagulation might also be useful when early serial measurements are performed. While we work on the question of potential new markers, Kim and colleagues have added insights to help answer the questions of when to measure and how to analyze clinically available markers of sepsis.

Conflicts of interest Corsino Rey has received speaker honoraria from Brahms and Thermofisher Companies to attend meetings related to sepsis biomarkers. Dr. Fortenberry reports no potential conflicts of interest.

\section{References}

1. Kaplan J, Wong H (2011) Biomarker discovery and development in pediatric critical care medicine. Pediatr Crit Care Med 12:165-173

2. Xu XJ, Tang YM, Liao C et al (2013) Inflammatory cytokine measurement quickly discriminates gram-negative from gram-positive bacteremia in pediatric hematology/oncology patients with septic shock. Intensive Care Med 39:319-326

3. Dellinger RP, Levy MM, Rhodes A et al (2013) Surviving Sepsis Campaign: international guidelines for management of severe sepsis and septic shock, 2012. Intensive Care Med 39:165-228

4. Bakker J, Gris P, Coffernils M, Kahn R, Vincent J-L (1996) Serial blood lactate levels can predict the development of multiple organ failure following septic shock. Am J Surg 171:221-226

5. Duke TD, Butt W, South M (1997) Predictors of mortality and multiple organ failure in children with sepsis. Intensive Care Med 23:684-692

6. Dugas MA, Proulx F, de Jaeger A, Lacroix J, Lambert M (2000) Markers of tissue hypoperfusion in pediatric septic shock. Intensive Care Med 26:75-83
7. Kim Y, Ha E-J, Jhang WK, Park SJ (2013) Early blood lactate area as a prognostic marker in pediatric septic shock. Intensive Care Med. doi:10.1007/s00134-013-2959-z

8. Yo Chia-Hung, Hsieh Pei-Shan, Lee SiHuei (2012) Comparison of the test characteristics of procalcitonin to C-reactive protein and leukocytosis for the detection of serious bacterial infections in children presenting with fever without source: a systematic review and meta-analysis. Ann Emerg Med 60:591-600

9. Rey C, Los Arcos M, Concha A et al (2007) Procalcitonin and C-reactive protein as markers of systemic inflammatory response syndrome severity in critically ill children. Intensive Care Med 33:477-484

10. Esposito S, Tagliabue C, Picciolli I et al (2001) Procalcitonin measurements for guiding antibiotic treatment in pediatric pneumonia. Respir Med 105:1939-1945

11. Wong HR, Cvijanovich N, Wheeler DS et al (2008) Interleukin-8 as a stratification tool for interventional trials involving pediatric septic shock. Am J Respir Crit Care Med 178:276-282
12. Lee JH, Chan YH, Lai OF, Puthucheary J (2013) Vasopressin and copeptin levels in children with sepsis and septic shock. Intensive Care Med 39:747-753

13. Massion PB, Peters P, Ledoux D et al (2012) Persistent hypocoagulability in patients with septic shock predicts greater hospital mortality: impact of impaired thrombin generation. Intensive Care Med 38:1326-1335

14. Marshall J, Reinhart K, International Sepsis Forum (2009) Biomarkers of sepsis. Crit Care Med 37:2290-2298

15. Mickiewicz B, Vogel HJ, Wong HR, Winston BW (2013) Metabolomics as a novel approach for early diagnosis of pediatric septic shock and its mortality. Am J Respir Crit Care Med 187:967-976 